\title{
Callous-unemotional traits, low cortisol reactivity and physical aggression in children: findings from the Wirral Child Health and Development Study
}

\author{
Nicola Wright [1', Jonathan Hill', Andrew Pickles ${ }^{1}$ and Helen Sharp ${ }^{3}$
}

\begin{abstract}
Callous-unemotional (CU) traits are thought to confer risk for aggression via reduced amygdala responsivity to distress cues in others. Low cortisol reactivity is thought to confer risk for aggression via reduced arousal and this effect may be confined to boys. We tested the hypothesis that the association between childhood CU traits and aggression would be greatest in the absence of the inhibitory effects of cortisol reactivity, and that this effect would be sex dependent. Participants were 283 members of a stratified subsample within an epidemiological longitudinal cohort (WCHADS). Cortisol reactivity to a social stressor was assessed at 5 years. CU traits were reported by mothers at 5 years, and physical aggression by mothers and teachers at age 7. Results showed that CU traits were associated with elevated aggression at 7 years controlling for earlier aggression. There was no main effect of cortisol reactivity on regression. The association between CU traits and aggression was moderated by cortisol reactivity $(p=.011)$ with a strong association between CU traits and aggression in the presence of low reactivity, and a small and non-significant association in the presence of high reactivity. This association was further moderated by child sex $(p=.041)$ with the joint effect of high CU traits and low cortisol reactivity seen only in boys $(p=.016)$. We report first evidence that a combined deficit in inhibitory processes associated with CU traits and low cortisol reactivity increases risk for childhood aggression, in a sex-dependent manner.
\end{abstract}

\section{Introduction}

Neurobiological models of persistent antisocial behaviours in children propose a prominent role for low physiological arousal leading to failures to inhibit aggressive behaviour, greater sensation seeking, and reduced effects of punishment ${ }^{1,2}$. Callous-unemotional (CU) traits are similarly thought to be associated with failures to inhibit aggression and reduced punishment learning, via mechanisms that implicate reduced

Correspondence: Nicola Wright (nwright@liv.ac.uk)

${ }^{1}$ Biostatistics Department at the Institute of Psychiatry, King's College London, London, UK

${ }^{2}$ School of Psychology and Clinical Language Sciences, University of Reading, Reading, UK

Full list of author information is available at the end of the article. amygdala activation to distress in others ${ }^{3,4}$. No previous study has examined the roles of both $\mathrm{CU}$ traits and reduced physiological arousal indexed by hypothalamicpituitary-adrenal (HPA) axis activity in the generation of childhood aggression. Further, the effects may differ in males and females, with failures of inhibition contributing to aggression in boys, and heightened reactivity in girls ${ }^{5-10}$. Using cortisol reactivity to hearing a recorded argument between adults as the index of physiological arousal, we set out to test the hypothesis that the association between $\mathrm{CU}$ traits and aggression would be strengthened in the presence of low cortisol reactivity in boys but not girls ${ }^{11,12}$.

Several mechanisms have been proposed linking underarousal to aggressive and antisocial behaviours notably via

\section{(c) The Author(s) 2019}

(c) (i) Open Access This article is licensed under a Creative Commons Attribution 4.0 International License, which permits use, sharing, adaptation, distribution and reproduction cc) in any medium or format, as long as you give appropriate credit to the original author(s) and the source, provide a link to the Creative Commons license, and indicate if changes were made. The images or other third party material in this article are included in the article's Creative Commons license, unless indicated otherwise in a credit line to the material. If material is not included in the article's Creative Commons license and your intended use is not permitted by statutory regulation or exceeds the permitted use, you will need to obtain permission directly from the copyright holder. To view a copy of this license, visit http://creativecommons.org/licenses/by/4.0/. 
sensation-seeking and risk-taking behaviours ${ }^{13}$, and fearlessness with reduced inhibition of aggression ${ }^{14,15}$. These models would lead to the prediction that reduced HPA axis reactivity will be associated with more behaviour problems. Current evidence is however inconsistent perhaps explained in part by marked heterogeneity of child ages, study designs, methods for assessing cortisol, and symptom measurement ${ }^{16}$. The majority of studies of HPA axis functioning have examined contributions to broad externalising problems. However, biological mechanisms may vary within this broad phenotype. In recent years the construct of $\mathrm{CU}$ traits has proved robust and informative at identifying a subgroup of antisocial children who show more severe and persistent antisocial behaviour ${ }^{17}$. Thus there may be a distinct aetiology for antisocial behaviour, and in particular for physical aggression, associated with $\mathrm{CU}$ traits compared to antisocial behaviour without $\mathrm{CU}$ traits $^{18,19}$. Deficits in recognition of fear and sadness, possibly related to lack of eye contact in social interactions, are thought to underpin indifference to others' distress in CU traits, and hence to children's failures to inhibit aggressive or cruel actions ${ }^{3,20,21}$.

Thus according to both the underarousal and CU traits hypotheses antisocial behaviours arise from failures in inhibitory mechanisms, and a lack of responsiveness to others' behaviours, either punishment or to emotional distress. The greatest risk for antisocial behaviours, and in particular for physical aggression, may therefore arise from the combination of the two. Neurobiological models of $\mathrm{CU}$ traits implicate lack of responsiveness of the amygdala to emotional cues which would typically serve to inhibit antisocial behaviour ${ }^{21,22}$, with the possibility of reciprocal effects between HPA axis regulation and amygdala function ${ }^{23,24}$. These considerations led Hawes et al. to speculate that, "...high levels of $\mathrm{CU}$ traits and HPA-axis hypoactivity characterise a particularly severe subgroup." Whether processes associated with CU traits and HPA axis hypo-activity are sufficiently distinct to make independent contributions to antisocial behaviours is not yet clear. Indeed it has been proposed that HPA axis hypoactivity contributes to $\mathrm{CU}$ traits, or that they are separate markers of the same underlying processes. However, studies examining whether $\mathrm{CU}$ traits are associated with HPA axis hyporeactivity have yielded inconsistent findings, and no studies have provided evidence to link reduced cortisol reactivity to $\mathrm{CU}$ traits outside of clinically referred samples ${ }^{25}$.

It has previously been proposed that the under-arousal pathway to antisocial behaviour problems may be characteristic of their development in boys but not in girls ${ }^{12}$. Increasingly there is evidence for sex differences in HPA axis mechanisms for antisocial behaviour in children, consistent with this hypothesis. This has been demonstrated in cross section and over time in a study 1768 children aged $10-12^{5,7}$, and in further cross-sectional studies of 245 adolescents $^{9}$ and 501 adolescents $^{26}$. In a longitudinal study of 283 children over the ages 6-9 more blunted cortisol rhythms at age 6 (less change across the day from morning to evening) predicted a greater increase in conduct problems and aggressive behaviour, more in boys than in girls ${ }^{27}$.

In summary, and with caveats arising from inconstancies in approaches and findings, available evidence is consistent with there being a pathway to aggression characterised by physiological underarousal and by lack of distress responsivity, which is more typical of boys than girls. In line with Hawes' et al. speculation we predicted the association between $\mathrm{CU}$ traits and aggression will be greatest in the presence of low cortisol reactivity, and that that this would be seen in boys but not in girls. We tested these predictions prospectively in community-based sample of children, with cortisol reactivity and CU traits assessed at 5 years, and child aggression at age 7 years.

\section{Method \\ Sample}

Participants were members of the Wirral Child Health and Development Study (WCHADS), a prospective epidemiological study starting in pregnancy. Ethical approval was granted by the Cheshire North and West Research Ethics committee on the 27 June 2006 (pregnancy to age 1 waves), 7 June 2010 (age 2.5-5 year waves), and 22 December 2014 (age 7 and 9 year waves). All women gave written informed consent at the point of recruitment in the antenatal clinic. The study used a two stage stratified design in which a consecutive general population sample (the 'extensive' sample) is used to generate a smaller 'intensive' sample stratified by psychosocial risk with more detailed measurement over time and both are followed in tandem ${ }^{28,29}$. [Further information about the data and conditions for access are available at the University of Liverpool Research Data Catalogue: https://doi.org/ 10.17638/datacat.liverpool.ac.uk/564.]

The whole cohort (extensive sample) comprised 1233 women recruited in pregnancy with a live, singleton baby for long-term follow-up post-birth (see Sharp et al. ${ }^{28}$ for detailed account of sampling). The mean age at recruitment was 26.8 years $(\mathrm{SD}=5.8$, range $18-51), 41.8 \%$ of the sample were in the most deprived quintile of UK neighbourhoods $^{30}$ and $96.1 \%$ were White British. There were 316 mothers recruited to the intensive sample at 32 weeks pregnancy and available at birth for longitudinal followup. At age 5 a further stratum was added to the intensive sample using established thresholds for emotional or behavioural symptoms in the Child Behaviour Checklist $\left(\mathrm{CBCL}^{31}\right)$ and the Antisocial Process Screening Device $\left(\mathrm{APSD}^{32,33}\right)$ used with the extensive sample at age 3.5 years. This identified 94 children of whom 75 (79.8\%) 
agreed and completed the age 5 assessment. This process yielded an intensive sample of 330 at age 5, of whom 314 (90\%) provided complete cortisol data. Eight cases were subsequently excluded; five had supra-physiological levels of cortisol and three had eaten or drunk prior to the final sample being taken.

In the analyses that follow, data from the larger extensive sample assessed at $7(n=778)$ were used to first estimate the aggression latent variable outcome variable from the mother $(n=769)$ and teacher reports $(n=725)$. The main analyses then used data from the intensive sample comprising 283 cases (of the 330 available from age 5) who provided data at both age 5 and 7 years. The mean age of this sample at the age 5 assessment was 57.59 months ( $\mathrm{SD}=2.44$, range 54-69) and at the age 7 assessment was 88.19 months (SD = 3.75 , range $=83-107)$ with slightly more boys $(n=145)$ than girls $(n=138)$. Of these $283,15.9 \%(n=45)$ of children (62\% boys) showed clinically significantly externalising problems on the $\mathrm{CBCL}$ according to mother or teacher report.

\section{Code availability}

Analysis code is available from the first author.

\section{Procedures and measures}

\section{Age 5 procedures and measures}

At age 5 the families in the intensive sample completed a $2.5 \mathrm{~h}$ lab assessment within which the stress induction task was embedded. Because arrival at the lab may activate an inticipatory cortisol rise, Koss and Gunnar ${ }^{34}$ recommend a 30-40 min relaxation period prior to taking a baseline sample. In this study, it was not possible to ensure this period was relaxing because in order to complete all the assessments it was necessary to present a range of cognitive and emotion recognition tasks to the children during the first $40 \mathrm{~min}$. Therefore, to provide a more robust baseline we took saliva samples at 20 and $40 \mathrm{~min}$ and used an average of the two. The child was exposed to the stress induction paradigm followed by an emotionally neutral cognitive task, with the post-stress cortisol sample taken 20 min after onset of the argument. Researchers' ensured that the child had been awake and had not eaten or drank for the $30 \mathrm{~min}$ prior to the first sample. Mothers were briefed about the stress induction task in private and children were debriefed after the procedure.

\section{Stress induction paradigm}

The stress paradigm involved the child overhearing an audiotaped recording of an argument between two adults $^{35}$. The task been used in previous studies of respiritory sinus arrhythmia ${ }^{35-37}$ and galvanic skin respons ${ }^{35,38}$. Mothers were asked to wait behind a screen whilst the child remained in the lab with the researcher completing the Kiddie Connors Continuous Performance Task $^{39}$. The recorded conversation started playing $15 \mathrm{~s}$ into the task, after a few seconds the researcher informed the child that the sound was people speaking in the next room, the researcher then sat away from the child and busied themselves with paperwork for the remainder of the recording. The $7 \mathrm{~min}$ recording comprised $2 \mathrm{~min}$ in which two work colleagues could be heard chatting about benign topics, $2 \mathrm{~min}$ intense argument, $2 \mathrm{~min}$ unresolved anger, and $1 \mathrm{~min}$ verbal resolution.

\section{Salivary hormone assessment and enzyme immunoassay procedure}

Salivary cortisol was collected using cotton eye swabs; the swab was placed in the child's cheek by the researcher until it was fully wet. Three swabs were collected and placed in a Salametrics tube. Saliva samples were frozen and stored at $-20^{\circ} \mathrm{C}$ until analysis. After thawing, salivettes were centrifuged at $3000 \mathrm{rpm}$ for $5 \mathrm{~min}$, which resulted in a clear supernatant of low viscosity. Salivary concentrations were measured using commercially available chemiluminescence immunoassay with high sensitivity (IBL International, Hamburg, Germany). Sample and reagent handling was semi-automated using a liquid handling robot (Genesis, Tecan, Switzerland) and quality control samples of low, medium, and high cortisol concentrations were run on each microtiter plate assayed. The intra and interassay coefficients for cortisol were both below $8 \%$. The derived cortisol levels were winsorized and cortisol reactivity was assessed by calculating a difference score between the mean of the two baseline cortisol samples and the post-stressor sample.

Cortisol levels vary throughout the course of the day, however, as this investigation was part of a large-scale longitudinal study, where substantial number of participants were seen over a short period of time, it was necessary to assess during morning and afternoons, and hence unrealistic to conduct all the assessments at the same time of day. Time of first cortisol sample was at average 11:58 (SD 2:11 h) and ranged from 8:54 to 17:20. There was no association between cortisol reactivity and time of day of cortisol assessment, on the full sample $(r=$ $-.05, p=.390)$ nor in boys $(r=-.03, p=.700)$ or girls $(r$ $=-.07, p=.389$ ) separately. Steroid medication use is also known to affect cortisol levels. Information on current prescription and non-prescription medications usage was collected and medications were dichotomised into steroid-related versus non-steroid-related medication or no medication. Forty (14\%) participants had used steroidrelated medications within the last 2 weeks; 27 reported cortisol-based cream use, 12 inhaled steroid use and 1 oral tablet steroid use. There were no significant differences in cortisol reactivity between children who had used steroid medication and those who had not $(p=.358$ full sample, 
$p=.506$ girls, and $p=.738$ boys). Medication use significantly predicted higher baseline cortisol levels on the full sample $(t=-1.98, \mathrm{df}=280, p=.048$; no medication use mean $=7.04$, medication use mean $=8.77$ ) and in girls $(t=-3.56, \mathrm{df}=135, p<.001$; no medication use mean $=7.07$, medication use mean $=12.74$ ) but not in boys $(p=.695)$. To account for any potential confounding effects on cortisol reactivity we ran a linear regression predicting cortisol reactivity from time of day and steroid medication use and used the residual score for the main analysis.

\section{CU traits}

$\mathrm{CU}$ traits were assessed by mother-report at 5 years using a combination of items from the $\mathrm{APSD}^{30}$, the $\mathrm{CBCL}^{29}$, and the Strengths and Difficulties Questionnaire (SDQ; 40). All items are rated on a three-point scale. Items were selected based on inclusion in $\mathrm{CU}$ traits measures in other studies ${ }^{41-44}$. We have previously created $\mathrm{CU}$ traits latent factor scores on this sample at ages $2.5,3.5$, and 5 years ${ }^{45}$ by subjecting items to exploratory and confirmatory factor analyses in MPlus ${ }^{46}$. The age 5 measure comprises 13 items which are listed in Supplementary Table S1 together with the factor loadings. The derived $\mathrm{CU}$ traits measure shows improved internal consistency $(\alpha=.83)$ compared to the APSD alone $(\alpha$ $=.60$ ) and partial strong factorial invariance by sex.

\section{Aggression}

Aggression was assessed by mother-report on a fiveitem physical aggression questionnaire at ages 5 and 7 years ${ }^{47}$. The questionnaire consists of five items previously shown to yield aggression scores with stability from ages 17 to 29 months ${ }^{47}$. Each item is rated on a three-point scale. The items were subjected to a confirmatory factor analysis in Mplus and a factor score was extracted for analysis.

\section{Confounders}

Deprivation was assessed using the indices of multiple deprivation (IMD; 30) based on UK postal codes in which a binary variable, with $1=$ most deprived quintile of UK neighbours versus $0=$ all other quintiles, was used for analysis. To account for the stratification, variables indicating whether the family was high-risk or low-risk allocation to the intensive sample were also included as covariates.

\section{Analysis plan}

First, the age 7 years mother and teacher aggression items were modelled as a single latent variable using the gsem command in Stata version $14^{48,49}$. A factor score was extracted for all subsequent analysis. Bivariate associations were examined using Spearmans and polychoric correlations. The main analysis used multiple linear regression with predictors entered as a series of blocks using the nestreg command which provides a Wald test of whether the addition of each block produces a significant improvement in the model. The first block contained the confounding variables (including the two stratification variables) and the main effects of child sex, age 5 aggression and CU traits and cortisol reactivity was added in the second block. The two-way interaction term between $\mathrm{CU}$ traits and cortisol was added in the third block, and the three-way interaction at the third block, together with the other two-way interactions. All variables were standardised prior to creating interaction terms.

Interactions were further explored in two ways. First the margins command was used to test the association between $\mathrm{CU}$ traits and aggression at mean and $1 \mathrm{SD}$ above and below the mean levels of cortisol reactivity. This was done in order to find out whether the interaction arose in accordance with the prediction that the association between $\mathrm{CU}$ traits and aggression will be greatest in the presence of low cortisol reactivity. Secondly, to address two potential limitations of the interaction, namely that it might be evident only in the presence of an increase in cortisol from baseline to poststress, and that it may not be seen at the upper end of the $\mathrm{CU}$ traits distribution likely to be most relevant to clinical samples, we explored the linearity of the interaction of covariate $X$ with moderator $M$. This was done by rewriting the usual regression of the form $E[y]=a+b 1 X$ $+b 2 M+c X M$ into the equivalent form $E[y]=a+(b 1+$ $c M) X+b 2 M$. We then plotted the coefficient $(b 1+c M)$ with its $95 \%$ confidence envelope that assumed linearity. To examine non-linearity we estimated group-specific estimates of this coefficient for different levels of the moderator variables, the groups being defined by deciles of cortisol reactivity and the levels being the median values of these groups. Since these group-wise estimates are highly variable, being estimated on small samples, the figure also displays a fractional polynomial smooth through them. To account for confounders, a model with the linear interaction and confounder main effects was first fitted, and the models required for the figure fitted to the adjusted aggression score obtained by subtracting the estimated effects of confounders.

We checked the distribution of the residuals from the analysis of the regression scores. Plots suggested modest skew but the Cook-Weisberg test was clearly significant $(p<.001)$. Analyses were repeated with the three observations further than 3SD from the mean removed, and also with robust standard errors that are robust to heteroscedasticity. Both analyses left the pattern of significant effects unchanged. Since the analyses with robust 
standard errors are likely to be the most reproducible, it is these that we report. The regression models were also estimated with all variables entered sumulataneously and are presented in the Supplementary Tables 3 and 5. Power calculations for the original cohort were approved by the funder (MRC) following peer review.

\section{Results}

\section{Computation of physical aggression outcome}

The factor loadings for the mother and teacher aggression items on the single aggression latent variable are shown in Supplementary Table 2 for the extensive sample. A factor score was extracted for all subsequent analyses.

The descriptive statistics for the key study variables are presented in Table 1 for boys and girls separately. As can be seen in Table 1 mean cortisol levels were lower poststress than at the baselines, with levels decreasing from baseline one to baseline two. In the full sample, $30 \%$ of children $(n=86)$ showed a rise from baseline to poststress, with $29 \%$ of girls $(n=40)$ and $32 \%$ of boys $(n=46)$. Bivariate associations are presented in Table 2, for boys and girls separately. Cortisol reactivity showed no significant associations with $\mathrm{CU}$ traits or age 5 or 7 aggression. CU traits were associated with age 5 and age 7 aggression in both boys and girls. Mothers' younger age at first pregnancy and deprivation were associated with increased aggression and $\mathrm{CU}$ traits, underlining the importance of controlling for these variables in subsequent analyses.

\section{Multivariate analysis}

The results of the main analysis are presented in Table 3. CU traits at age 5 years predicted child aggression at age 7 years, controlling for age 5 years aggression, however there was no main effect of cortisol reactivity. The two-way interaction between $\mathrm{CU}$ traits and cortisol reactivity, introduced in the third block, was significant $(p$ $=.011$ ). The effect of the interaction is shown in Fig. 1 contrasting associations between $\mathrm{CU}$ traits at age 5 and aggression at age 7 at low (1 SD below mean), medium (mean), and high levels of reactivity (1 SD above mean). The association between $\mathrm{CU}$ traits and child aggression was greatest in association with low cortisol reactivity $(\beta$ $=.50, \mathrm{SE}=.12, p<.001)$ and progressively lessened at mean reactivity $(\beta=.36, \mathrm{SE}=.10, p<.001)$ and at high reactivity $(\beta=.22$, $\mathrm{SE}=.13, p=.084)$.

The three-way interaction between sex, cortisol reactivity, and CU traits was significant $(p=.041)$ reflecting that there was a two-way interaction in boys $(p=.016)$ but not in girls $(p=.799$; the full model coefficients are presented in Supplementary Table 4 and Supplementary Figure 1 presents the interaction, for boys and girls seperately). In boys, $\mathrm{CU}$ traits significantly predicted aggression at low reactivity $(\beta=.78, \mathrm{SE}=.18, p<.001)$ and at mean reactivity $(\beta=.49, \mathrm{SE}=.15, p=.001)$ but not at high reactivity $(\beta=.19, \mathrm{SE}=.19, p=.276)$.

Figure 2 plots the interaction at 10 deciles of cortisol reactivity. The figure indicates that the changing association between $\mathrm{CU}$ traits and aggression occurs across the distribution on reactivity and not among only those

Table 1 Descriptive statistics for the key study variables for boys and girls separately

\begin{tabular}{|c|c|c|c|c|}
\hline & \multicolumn{2}{|l|}{$\begin{array}{l}\text { Boys } \\
N=145\end{array}$} & \multicolumn{2}{|l|}{$\begin{array}{l}\text { Girls } \\
N=138\end{array}$} \\
\hline & Mean (SD) & Range & Mean (SD) & Range \\
\hline \multicolumn{5}{|l|}{ Age 7} \\
\hline Physical aggression (teacher and mother report) ${ }^{a}$ & $.68(2.05)$ & -.79 to 7.22 & $-.18(1.33)$ & -.79 to 5.31 \\
\hline \multicolumn{5}{|l|}{ Age 5} \\
\hline Cortisol baseline 1 & $7.34(4.19)$ & $1.03-26.56$ & $8.17(5.88)$ & $1.90-30.37$ \\
\hline Cortisol baseline 2 & $6.49(5.43)$ & $.59-33.68$ & $7.30(6.94)$ & $1.01-33.68$ \\
\hline Cortisol post-stressor & $5.67(4.99)$ & $.47-30.52$ & $6.68(5.87)$ & $1.49-30.52$ \\
\hline Aggression (mother report) ${ }^{\mathrm{a}}$ & $.45(.69)$ & -.03 to 1.81 & $.24(.56)$ & -.03 to 1.81 \\
\hline $\mathrm{CU}$ traits $^{\mathrm{a}}$ & $.13(.34)$ & -.51 to 1.20 & $.02(.34)$ & -.51 to 1.04 \\
\hline \multicolumn{5}{|l|}{ Confounding variables } \\
\hline Mothers age at pregnancy & $27.30(6.27)$ & $18-51$ & $27.66(5.94)$ & $18-41$ \\
\hline Most deprived ${ }^{\mathrm{b}}: n(\%)$ & $51(35.2)$ & $0-1$ & $56(40.1)$ & $0-1$ \\
\hline
\end{tabular}

${ }^{\mathrm{a}}$ Factor scores extracted from a latent variable

${ }^{\mathrm{b}}$ Most deprived $=$ in highest national quintile for deprivation 


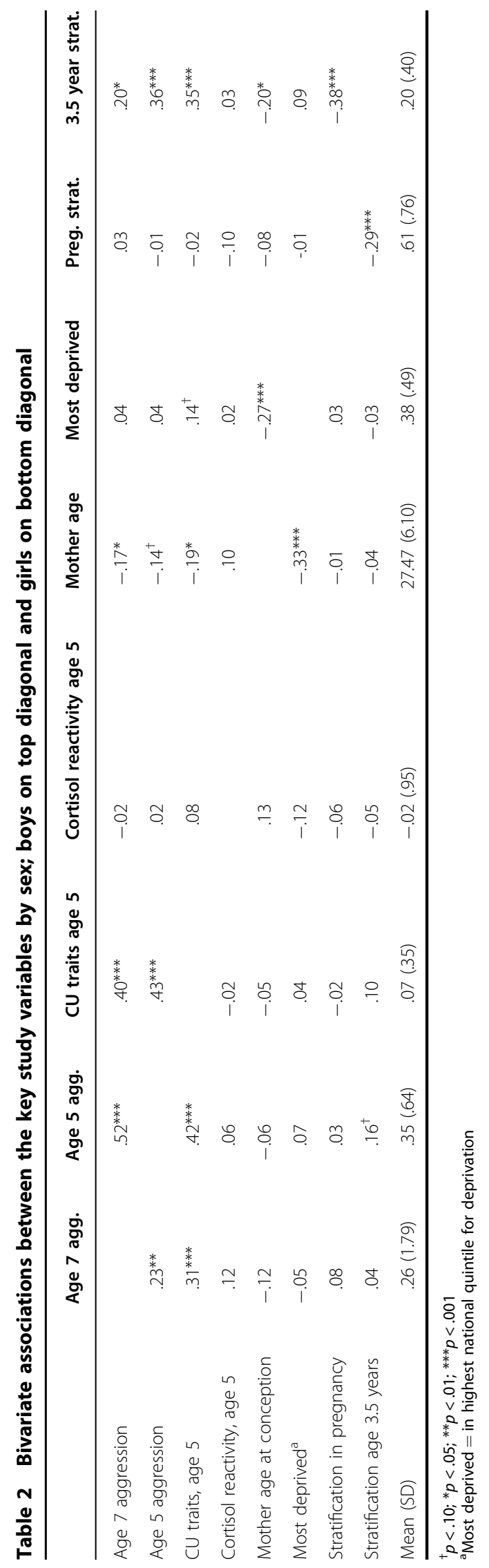

Table 3 Summary of linear regression model predicting age 7 aggression from age 5 cortisol reactivity, CU traits and child sex

\begin{tabular}{|c|c|c|}
\hline & $\beta$ & $p$ \\
\hline \multicolumn{3}{|l|}{ Block 1} \\
\hline Mothers age & -.11 & .056 \\
\hline Most deprived & -.07 & .195 \\
\hline $\begin{array}{l}\text { Sample stratification status: } \\
\text { pregnancy stratum } 1\end{array}$ & -.01 & .798 \\
\hline $\begin{array}{l}\text { Sample stratification status: } \\
\text { pregnancy stratum } 2\end{array}$ & .05 & .490 \\
\hline $\begin{array}{l}\text { Sample stratification status: } \\
3.5 \text { years }\end{array}$ & -.01 & .881 \\
\hline Child sex ${ }^{a}$ & -.14 & .006 \\
\hline Age 5 aggression & .32 & $p<.001$ \\
\hline \multirow[t]{2}{*}{ Age $5 \mathrm{CU}$ traits } & .21 & $p<.001$ \\
\hline & \multicolumn{2}{|c|}{$F(9,274)=8.98, p<.001, R^{2}=.27$} \\
\hline \multicolumn{3}{|l|}{ Block 2} \\
\hline \multirow[t]{2}{*}{ Cortisol reactivity } & .01 & .816 \\
\hline & \multicolumn{2}{|c|}{$F(1,282)=.05, p=.816, R^{2}=.27, R^{2} \Delta=.00$} \\
\hline \multicolumn{3}{|l|}{ Block 3} \\
\hline \multirow[t]{2}{*}{ CU traits*Cortisol reactivity } & -.11 & .011 \\
\hline & \multicolumn{2}{|c|}{$F(1,282)=6.57, p=.011, R^{2}=.28, R^{2} \Delta=.01$} \\
\hline \multicolumn{3}{|l|}{ Block 4} \\
\hline CU traits*Cortisol reactivity & -.37 & .007 \\
\hline Child sex*Cortisol reactivity & .05 & .758 \\
\hline Child sex* ${ }^{*} \mathrm{CU}$ traits & -.24 & .165 \\
\hline \multirow{2}{*}{$\begin{array}{l}\text { Child Sex*CU traits*Cortisol } \\
\text { reactivity }\end{array}$} & .27 & .041 \\
\hline & \multicolumn{2}{|c|}{$F(3,280)=2.07 p=.10, R^{2}=.29, R^{2} \Delta=.01$} \\
\hline
\end{tabular}

${ }^{\text {a }}$ Child sex coded as $1=$ male, $2=$ female

who showed a rise in cortisol after the stressor. Supplementary Figure 2 presents this plot in boys and girls separately; the boys plot largely mirrors that found on the full sample with the girls plot consistent with no moderation by cortisol reactivity. Supplementary Figures 3 and 4 plot the interaction at deciles of CU traits, on the whole sample and in boys and girls separately, respectively. The figures indicate that the interaction can be seen across the distribution of $\mathrm{CU}$ traits scores and is not restricted to high or low scorers.

\section{Discussion}

In a longitudinal general population sample with cortisol reactivity to a social stressor, $\mathrm{CU}$ traits and child aggression assessed at age 5 years, the highest levels of aggression at age 7 years were predicted by the combination of high CU traits and low cortisol reactivity. This is consistent with the hypothesis put forward by Hawes et al. ${ }^{11}$. The two-way interaction was seen across the sample, but the effect was further modified by sex of child. The three-way interaction arose because in girls there was an association between $\mathrm{CU}$ traits and later aggression that was similar at all levels of cortisol reactivity, while in boys the association was markedly different at low and high levels. This effect of CU traits and cortisol reactivity at age 


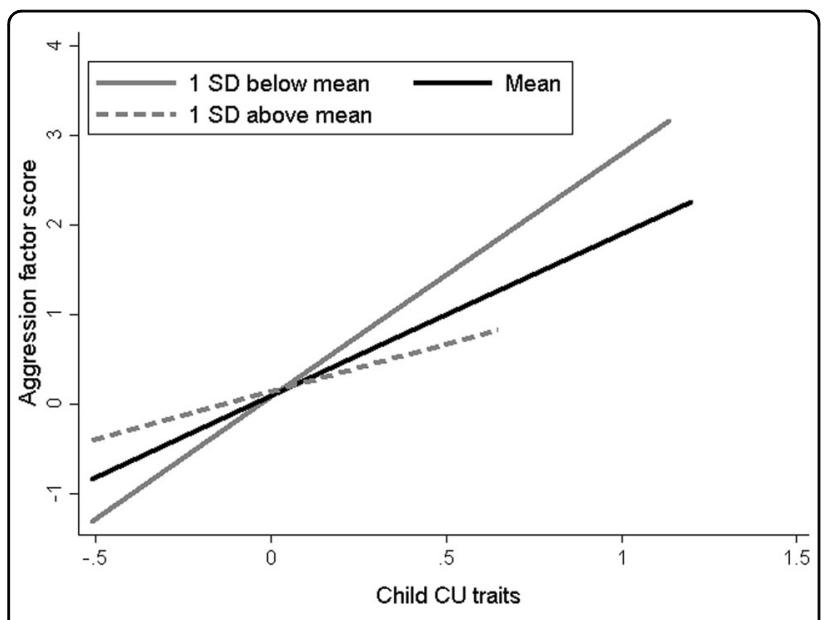

Fig. 1 The prospective association between CU traits and aggression at 'low' (1 SD below mean), 'medium' (mean) and 'high' (1 SD above mean) cortisol reactivity

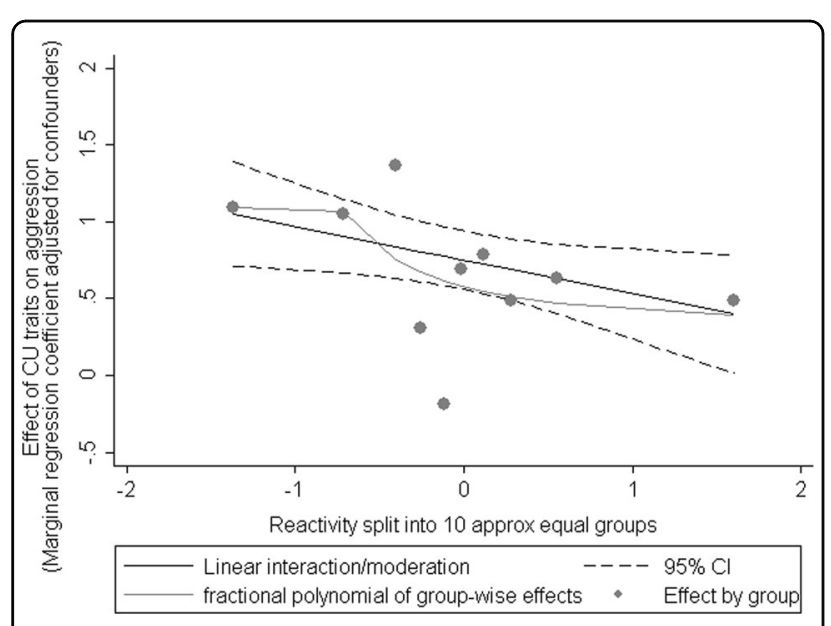

Fig. 2 The effect of CU traits on aggression at 10 deciles of cortisol reactivity (adjusted for confounders)

5 years on aggression at 7 years remained after controlling for child aggression at age 5 years.

Although the prediction of age 7 aggression from age 5 $\mathrm{CU}$ traits was not the main focus of the study, the finding of an association over and above the continuity between age 5 and age 7 aggression provided further support for a continuing effect of $\mathrm{CU}$ traits even after age 5 when child aggression has commonly become established. This is a well replicated effect in older children, consistent with growing evidence in young children ${ }^{6,50}$. The lack of main effect of cortisol reactivity on child aggression from age 5 to age 7 is consistent with previous findings. ${ }^{16}$.

While the significant interaction between $\mathrm{CU}$ traits and cortisol reactivity was seen across the sample as a whole, it was driven by the effect in boys, and was not seen at all in girls. This sex difference has been reported in previous studies of HPA-axis activity outlined earlier ${ }^{5,7,26,37}$. It is also consistent with other findings of physiogical reactivity and externalising problems in children. In relation to respiratory sinus arrhythmia (RSA), reductions to a stressor, used as an index of vagal reactivity, several studies have found externalising symptoms associated with decreased vagal reactivity in boys but increased reactivity in girls ${ }^{6-8,10}$. Twin studies of the aeteology of both $\mathrm{CU}$ traits and conduct problems have identified sex differences, in particular a greater shared environment influence for girls than boys ${ }^{51,52}$. Collectively this evidence suggests that there may be distinct aeteological pathways to the development of antisocial behaviour in boys and girls.

The study had a number of strengths. This was a prospective study of a consecutive sample from an antenatal clinic serving a defined geographical area. The subsample was created by stratifying for psychosocial risk during pregnancy and child symptoms at age 3.5 years and inclusion of the sample stratification factors in the models allowed generalisations to be made to the general population. Child CU traits and cortisol reactivity were assessed at age 5 and aggression was assessed at ages 5 and 7 . Both teacher and mother report of aggression were collected at age 7 and combined to produce a single latent variable outcome. This created a more robust aggression outcome which sampled behaviour in multiple domains, reduced the risk of inferential errors from multiple testing and also helped to reduce the effect of common method variance on the reporting of $\mathrm{CU}$ traits and aggression.

A key limitation of the study is that the experimental stressor for cortisol reactivity did not lead to an overall rise in cortisol levels. This is a widely reported finding in studies of cortisol reactivity in young children ${ }^{53}$. Within the context of a longitudinal study where retention of participants over a long period of time is paramount, higher levels of stress may have meant that children would not have wanted to remain in the study. In this sample we observed an overall decrease in cortisol levels from the first to second baseline and then to the post-stressor samples, consistent with an initial rise in response to arrival in the lab which reduced throughout the testing session. This would support the possibility that the response to the planned stressor was superimposed on falling cortisol following the first unplanned stressor on arrival at the lab. Whether or not variations in rates of fall following the planned stressor provide valid measures of an individual's reactivity, in the same way as variations in increases, is not known. As far as we are aware we are the first to have set out to examine this by assessing whether the cortisol reactivity by $\mathrm{CU}$ traits interaction varied across the distribution of reactivity scores, and in particular whether the effect was confined to the subgroup of children showing a pre-post stressor rise. There was 
convincing evidence that this was not the case supporting the validity of pre-post differences in cortisol even in the absence of an overall rise. This is consistent with findings from a number of other studies of young children that have yielded informative findings in the absence of overall effects on cortisol levels ${ }^{54-57}$, including prospective associations between cortisol reactivity to a lab stressor at age 3 years and externalising and internalising symptoms at age 6 years ${ }^{54}$.

The need to assess large numbers of children over a short period of time also meant that cortisol collections were not completed at the same time of day for all participants. However, this was accounted for by a correction for time of day in all analyses by creating a residualised cortisol score. The sample was recruited from a defined geographical area with a wide range of socioecomic conditions, but with very few non-white families, so the findings may not be not generalisable to other ethnic groups. This study used a community sample to investigate the processes involved in the translation of CU traits to aggressive behaviour, with only a minority of the sample showing clinically significant behavioural problems, and so it cannot be assumed that the findings generalise to clinical populations. However, we showed that the interaction between CU traits and cortisol reactivity could be seen across the distribution on CU traits scores, providing first evidence that these processes operate similarly in children with both high and low CU traits scores. Finally, we used a brief physical aggression measure which did not distinguish different forms of aggressive behaviour when developmental models of $\mathrm{CU}$ traits have placed emphasis on their role in proactive aggression $^{57}$.

The findings reported here were based on the hypothesis that both reduced amygdala reactivity to others' emotions and hence lower empathy, and reduced arousal and hence reduced inhibition, would jointly contribute to risk for aggression. While the interactions that we found may reflect such a synergy, other explanations are possible. Nevertheless they suggest that further investigation of the role of amygdala reactivity together with HPA axis reactivity would add to our understanding of mechanisms in male aggression. Whether or not there are different mechanisms in girls also requires further study.

\footnotetext{
Acknowledgements

The research was funded by grants from the UK Medical Research Council (G0900654; G0400577; MR/L022257/1). The authors are grateful to all participating families and research staff who contributed to this work. They also thank Wirral University Teaching Hospital NHS Foundation Trust, Wirral Community NHS Foundation Trust, Cheshire and Wirral Partnership NHS Foundation Trust and the National Institute for Health Research (NIHR) Biomedical Research Centre at South London and Maudsley NHS Foundation Trust.
}

\section{Author details}

${ }^{1}$ Biostatistics Department at the Institute of Psychiatry, King's College London, London, UK. ${ }^{2}$ School of Psychology and Clinical Language Sciences, University of Reading, Reading, UK. ${ }^{3}$ Department of Psychological Science, Institute of Psychology, Health and Society, University of Liverpool, Liverpool, UK

\section{Conflict of interest}

The authors declare that they have no conflict of interest.

\section{Publisher's note}

Springer Nature remains neutral with regard to jurisdictional claims in published maps and institutional affiliations.

Supplementary Information accompanies this paper at (https://doi.org/ 10.1038/s41398-019-0406-9).

Received: 11 September 2018 Revised: 27 November 2018 Accepted: 2 January 2019

Published online: 11 February 2019

\section{References}

1. Raine, A., Brennan, P. A. \& Farrington D. P. Biosocial bases of violence: conceptual and theoretical issues. In Biosocial Bases of Violence (eds., Raine, A., Brennan, P. A., Farrington, D. P. \& Mednick, S. A.) 1-20 (Plenum, New York, 1997).

2. Van Goozen, S. H., Fairchild, G., Snoek, H. \& Harold, G. T. The evidence for a neurobiological model of childhood antisocial behavior. Psychol. Bull. 133, 149 (2007).

3. Blair, R. J. Neurocognitive models of aggression, the antisocial personality disorders, and psychopathy. J. Neurol. Neurosurg. Psychiatry 71, 727-731 (2001).

4. Patrick, C. J. Emotion and psychopathy: startling new insights. Psychophysiology 1, 319-330 (1994)

5. Dietrich, A. et al. Cortisol in the morning and dimensions of anxiety, depression, and aggression in children from a general population and clinicreferred cohort: an integrated analysis. the TRAlLS study. Psychoneuroendocrinology 38, 1281-1298 (2013).

6. Hinnant, J. B. \& El-Sheikh, M. Codevelopment of externalizing and internalizing symptoms in middle to late childhood: sex, baseline respiratory sinus arrhythmia, and respiratory sinus arrhythmia reactivity as predictors. Dev. Psychopathol. 25, 419-436 (2013).

7. Marsman, R. et al. HPA-axis activity and externalizing behavior problems in early adolescents from the general population: the role of comorbidity and gender-the TRAILS study. Psychoneuroendocrinology 33, 789-798 (2008).

8. Morales, S., Beekman, C., Blandon, A. Y., Stifter, C. A. \& Buss, K. A. Longitudinal associations between temperament and socioemotional outcomes in young children: the moderating role of RSA and gender. Dev. Psychobiol. 57, 105-119 (2015).

9. Poustka, L. et al. Negative association between plasma cortisol levels and aggression in a high-risk community sample of adolescents. J. Neural Transm. 117, 621-627 (2010).

10. Vidal-Ribas, P., Pickles, A., Tibu, F., Sharp, H. \& Hill, J. Sex differences in the associations between vagal reactivity and oppositional defiant disorder symptoms. J. Child Psychol. Psychiatry 58, 988-997 (2017).

11. Hawes, D. J., Brennan, J. \& Dadds, M. R. Cortisol, callous-unemotional traits, and pathways to antisocial behavior. Curr. Opin. Psychiatry 22, 357-362 (2009).

12. Keenan, K., Shaw, D. Starting at the beginning: exploring the aetiology of antisocial behaviour in the first years of life. In Causes of Conduct Disorder and Juvenile Delinquency (eds. Farrington, D., Loeber, R.) 153-181 (Guilford Press, Guilford, 2003).

13. Zuckerman, M. Sensation Seeking: Beyond the Optimum Level of Arousal. (Erlbaum, Hillsdale, NJ, 1979).

14. Blair, R. J. Responsiveness to distress cues in the child with psychopathic tendencies. Pers. Individ. Dif. 27, 135-145 (1999).

15. Raine, A. Autonomic nervous system activity and violence (eds D. M, Stoff. \& R. B, Cairns.) Aggression and Violence 145-168 (Erlbaum: Mahwah, NJ, 1996).

16. Alink, L. R. A. et al. Cortisol and externalizing behavior in children and adolescents: Mixed meta-analytic evidence for the inverse relation of basal cortisol 
and cortisol reactivity with externalizing behavior. Dev. Psychobiol. 50, 427-450 (2008).

17. Frick, P. J., Ray, J. V., Thornton, L. C. \& Kahn, R. E. Can callous-unemotional traits enhance the understanding, diagnosis, and treatment of serious conduct problems in children and adolescents? A comprehensive review. Psychol. Bull. 140, 1 (2014).

18. Dadds, M. R. \& Rhodes, T. Aggression in young children with concurrent callous-unemotional traits: can the neurosciences inform progress and innovation in treatment approaches? Philos. Trans. R. Soc. L B Biol. Sci. $\mathbf{3 6 3}$, 2567-2576 (2008).

19. Frick, P. J. \& Morris, A. S. Temperament and developmental pathways to conduct problems. J. Clin. Child Adolesc. Psychol. 33, 54-68 (2004).

20. Dadds, M. R., Jambrak, J., Pasalich, D., Hawes, D. J. \& Brennan, J. Impaired attention to the eyes of attachment figures and the developmental origins of psychopathy. J. Child Psychol. Psychiatry 52, 238-245 (2011).

21. Blair, R. J. R. The neurobiology of psychopathic traits in youths. Nat. Rev. Neurosci. 14, 786-799 (2013).

22. Blair, R. J. The amygdala and ventromedial prefrontal cortex in morality and psychopathy. Trends Cogn. Sci. 11, 387-392 (2007).

23. Moriceau, S., Wilson, D. A., Levine, S. \& Sullivan, R. M. Dual circuitry for odor-shock conditioning during infancy: corticosterone switches between fear and attraction via amygdala. J. Neurosci. 26, 6737-6748 (2006).

24. Taylor, S. E. et al. Neural bases of moderation of cortisol stress responses by psychosocial resources. J. Pers. Soc. Psychol. 95, 197 (2008).

25. Moul, C., Hawes, D. J. \& Dadds, M. R. Mapping the developmental pathways of child conduct problems through the neurobiology of empathy. Neurosci. Biobehav. Rev. 91, 34-50 (2018).

26. Young, R., Sweeting, H. \& West, P. Associations between DSM-IV diagnosis, psychiatric symptoms and morning cortisol levels in a community sample of adolescents. Soc. Psychiatr. Psychiatr. Epidemiol. 47, 723-733 (2012).

27. Salis, K. L., Bernard, K., Black, S. R., Dougherty, L. R. \& Klein, D. (2016): Examining the concurrent and longitudinal relationship between diurnal cortisol rhythms and conduct problems during childhood. Psychoneuroendocrinology 71, 147-154 (2016).

28. Sharp, H. et al. (2012): Frequency of infant stroking reported by mothers moderates the effect of prenatal depression on infant behavioural and physiological outcomes. PLOS ONE 7, e45446 (2012).

29. Moffitt, T. E. et al. Do partners agree about abuse in their relationship?: a psychometric evaluation of interpartner agreement. Psychol. Assess. 9, 47-56 (1997).

30. Noble, M. et al. The English Indices of Deprivation 2004 (revised). Report to the Office of the Deputy Prime Minister (Neighbourhood Renewal Unit, London, 2004).

31. Achenbach, T. M. \& Rescorla, L. A. Manual for the ASEBA Preschool Forms \& Profiles. (University of Vermont, Research Center for Children, Youth and Families, Burlington, VT, 2000).

32. Frick, P. J. \& Hare, R. D. The Antisocial Process Screening Device (APSD). (MultiHealth Systems, Toronto, 2001).

33. Barry, C. T. et al. The importance of callous-unemotional traits for extending the concept of psychopathy to children. J. Abnorm. Psychol. 109, 335-340 (2000).

34. Koss, K. J. \& Gunnar, M. R. Annual research review: early adversity, the hypothalamic-pituitary-adrenocortical axis, and child psychopathology. J. Child Psychol. Psychiatry 59, 327-346 (2018).

35. Pollak, S. D., Vardi, S., Putzer Bechner, A. M. \& Curtin, J. J. Physically abused children's regulation of attention in response to hostility. Child Dev. 76, 968-977 (2005).

36. El-Sheikh, M., Harger, J. \& Whitson, S. M. Exposure to interparental conflict and children's adjustment and physical health: the moderating role of vagal tone. Child Dev. 72, 1617-1636 (2001).

37. Hinnant, J. B. \& El-Sheikh, M. Codevelopment of externalizing and internalizing symptoms in middle to late childhood: sex, baseline respiratory sinus arrhythmia, and respiratory sinus arrhythmia reactivity as predictors. Dev. Psychopathol. 25, 419-436 (2013).

38. El-Sheikh, M., Erath, S. A., Buckhalt, J. A., Granger, D. A. \& Mize, J. Cortisol and children's adjustment: the moderating role of sympathetic nervous system activity. J. Abnorm. Child Psychol. 36, 601-611 (2008).

39. Conners, C. K. \& Staff, M. Conners' Kiddie Continuous PerformanceTest (K-CPT). Computer Program for Windows Technical Guide and Software Manual. (MultiHealth Systems, Inc, Toronto, ON, 2001).

40. Goodman, R. The Strengths and Difficulties Questionnaire: a research note. J. Child Psychol. Psychiatry 38, 581-586 (1997).

41. Dadds, M. R., Hawes, D. J., Frost, A. \& Fraser, J. (2005): Disentangling the underlying dimensions of psychopathy and conduct problems in childhood: a community study. J. Consult. Clin. Psychol. 73, 400-410 (2005).

42. Hyde, L. W. et al. Dimensions of callousness in early childhood: links to problem behavior and family intervention effectiveness. Dev. Psychopathol. 25, 347-363 (2013).

43. Kimonis, E. R., Bagner, D. M., Linares, D., Blake, C. A. \& Rodriguez, G. Parent training outcomes among young children with callous-unemotional conduct problems with or at risk for developmental delay. J. Child Fam. Study 23, 437-448 (2014).

44. Willoughby, M. T., Waschbusch, D. A., Moore, G. A. \& Propper, C. B. Using the ASEBA to screen for callous unemotional traits in early childhood: factor structure, temporal stability, and utility. J. Psychopathol. Behav. 33, 19-30 (2011).

45. Wright, N., Hill, J., Sharp, H. \& Pickles, A. Maternal sensitivity to distress, attachment and the development of callous-unemotional traits in young children. J. Child Psychol. Psychiatry 59, 790-800 (2018).

46. Muthén, L. K. \& Muthén, B. O. Mplus User's Guide. 7th edn, (Muthén \& Muthén, Los Angeles, CA, 2012).

47. Baillargeon, R. H. et al. Gender differences in physical aggression: a prospective population-based survey of children before and after 2 years of age. Dev. Psychol. 43, 13-26 (2007).

48. StataCorp. Stata Statistical Software: Release, Vol. 14. (StataCorp LP, College Station, TX, 2015).

49. Waller, R., Hyde, L. W., Grabell, A. S., Alves, M. L. \& Olson, S. L. Differential associations of early callous-unemotional, oppositional, and ADHD behaviors: multiple domains within early-starting conduct problems? J. Child Psychol. Psychiatry 56, 657-666 (2015).

50. Waller, R. et al. Factor structure and construct validity of the parent-reported Inventory of Callous-Unemotional Traits among high-risk 9-year-olds. Assessment 22, 561-580 (2015).

51. Fontaine, N. M., Rijsdijk, F. V., McCrory, E. J. \& Viding, E. Etiology of different developmental trajectories of callous-unemotional traits. J. Am. Acad. Child Adolesc. Psychiatry 49, 656-664 (2010).

52. Meier, M. H., Slutske, W. S., Heath, A. C. \& Martin, N. G. Sex differences in the genetic and environmental influences on childhood conduct disorder and adult antisocial behavior. J. Abnorm. Psychol. 120, 377 (2011).

53. Gunnar, M. R., Talge, N. M. \& Herrera, A. Stressor paradigms in developmental studies: What does and does not work to produce mean increases in salivary cortisol. Psychoneuroendocrinology 34, 953-967 (2009).

54. Barrios, C. S., Bufferd, S. J., Klein, D. N. \& Dougherty, L. R. The interaction between parenting and children's cortisol reactivity at age 3 predicts increases in children's internalizing and externalizing symptoms at age 6. Dev. Psychopathol. 29, 1319-1331 (2017).

55. Ouellet-Morin, I. et al. Variations in heritability of cortisol reactivity to stress as a function of early familial adversity among 19-month-old twins. Arch. Gen. Psychiatry 65, 211-218 (2008).

56. Steptoe, A., van Jaarsveld, C. H., Semmler, C., Plomin, R. \& Wardle, J. Heritability of daytime cortisol levels and cortisol reactivity in children. Psychoneuroendocrinology 34, 273-280 (2009).

57. Frick, P. J. \& Morris, A. S. Temperament and developmental pathways to conduct problems. J. Clin. Child Adol. Psychol. 33, 54-68 (2004). 